\title{
Die nasslaufende Kupplung als Stellglied zur Schwingungsreduzierung im Antriebsstrang - Einflüsse eines veränderten Tribosystems
}

\author{
Arne Bischofberger ${ }^{1} \cdot$ Sascha Ott $^{1} \cdot$ Albert Albers $^{1}$ \\ Eingegangen: 29. Oktober 2019 / Angenommen: 26. November 2020 / Online publiziert: 14. Dezember 2020 \\ (c) Der/die Autor(en) 2020
}

\section{Zusammenfassung}

Eine Veränderung der Systemstruktur (tribologisches System) kann die Schwingungsreduzierung im nasslaufenden Kupplungssystem bei ansonsten gleichbleibenden Randbedingungen möglicherweise maßgeblich verbessern. Dieser Beitrag stellt hierzu Untersuchungen bei gezielter Variation des Tribosystems, im Speziellen des Reibbelages und des verwendeten Öls, vor. Für eine relative Bewertung der Schwingungsentkopplung werden die Bewertungsgrößen spezifisches Amplitudenverhältnis und die Winkelbeschleunigung eingeführt und herangezogen. Zusätzlich wird das dynamische Reibungszahlverhalten der Tribosystemvarianten gegenübergestellt und auf mögliche Korrelationen mit dem Schwingungsentkopplungsverhalten hin untersucht.

\section{Wet running clutch as actuator for reduction of oscillation in the power train-Influences of the tribological system}

\begin{abstract}
A variation of the tribological system can potentially significantly improve the decoupling behavior of the wet running clutch system. This paper presents investigations on specific variation of the tribological system, in particular the friction lining as well as the cooling oil. For a relative analyses of the vibration reduction, the evaluation parameters specific amplitude ratio and angular acceleration are introduced and used. Additionally the dynamic friction coefficient behavior is compared. Thereby possible correlations with the decoupling behavior are analysed.
\end{abstract}

\section{Einleitung und Stand der Forschung}

Effizienzsteigernde Maßnahmen in Kraftfahrzeugen wie u. a. Downsizing- und Downspeeding bringen mitunter unerwünschte Nebeneffekte wie höhere Momentenanregungen durch die Antriebsmaschine mit sich. Die gleichzeitig weiter zunehmenden Ansprüche an Komfort durch den Kunden fordern neue Maßnahmen zur Schwingungsreduzierung im modernen Antriebsstrang. Durch gezielt einstellbaren Kupplungsschlupf bietet das moderne nasslaufende Kupplungssystem die Möglichkeit Drehungleichförmigkeiten im Antriebsstrang zu reduzieren. Dieses Potenzial der Kupplung wurde bereits in [1] und [2] beschrieben.

Arne Bischofberger

arne.bischofberger@kit.edu

1 IPEK - Institut für Produktentwicklung, Karlsruher Institut für Technologie (KIT), Karlsruhe, Deutschland
Auch [3-5] gehen auf das Potenzial zur Drehschwingungsdämfpung durch geregelten Schlupfbetrieb ein [6]. betont die Vorteile einer schlupfgeregelten Kupplung zur Reduzierung von Torsionsschwingungen in Bereichen geringerer Drehzahl und gleichzeitig erhöhter Motorlast. Diese Funktionserweiterung der Kupplung bietet die Möglichkeit in zukünftigen u. a. hybriden Antriebssträngen andere schwingungsreduzierende Antriebsstrangkomponenten in Bauraum und Masse kleiner dimensionieren oder gänzlich außen vor lassen zu können.

In [7] wurde die Schwingungsreduzierung von Drehungleichförmigkeiten durch das Kupplungssystem im geregelten Dauerschlupfzustand aufgegriffen und simulativ bestätigt. Dabei wurde auch festgehalten, dass das Übertragungsverhalten im nasslaufenden Lamellenpaket maßgeblich vom Reibverhalten im Friktionskontakt bestimmt wird. Um hieran anknüpfend experimentelle Untersuchungen zu ermöglichen, wurde die in [8] vorgestellte Validierungsumgebung entwickelt. Die Validierungsumgebung bietet 
die Möglichkeit zur dynamischen Untersuchung des Reibungszahl- sowie des Schwingungsentkopplungsverhaltens im nasslaufenden Lamellenpaket unter gezielt aufgebrachter torsionaler Anregung. Erste Untersuchungen bestätigen das Potenzial zur Schwingungsreduzierung. Experimentelle Untersuchungen zum dynamischen Reibverhalten in einer geregelten Wandlerüberbrückungskupplung wurden in [9] durchgeführt. Auch in [10] wurden experimentelle, schwingungstechnische Untersuchungen in nasslaufenden Reibschaltelementen durchgeführt. Der Fokus lag in beiden Untersuchungen auf selbsterregten Reibschwingungen. In [11] und [12] wurde dagegen der Einfluss von Beanspruchungsgrößen auf das dynamische Reibverhalten im nasslaufenden Friktionskontakt unter gezielt aufgebrachter dynamischer Anregung untersucht. Außerdem wurde der Einfluss eines Fahrzeugrestsystems auf das Verhalten untersucht, wobei ein maßgeblicher Einfluss durch triebstrangdynamische Wechselwirkungen ermittelt werden konnte. In dynamischen Untersuchungen am Lamellenpaket ist deshalb das Fahrzeugrestsystem mit seinen schwingungsdynamischen Eigenschaften zu berücksichtigen [12]. Einflussgrößen auf die Schwingungsentkopplung wurden in [13] untersucht. Dabei wurden verschiedene Beanspruchungsgrößen, wie z.B. die Grundschlupfdrehzahl, die Flächenpressung oder der Kühlölvolumenstrom betrachtet. Es konnte ein maßgeblicher Einfluss der Grundschlupfdrehzahl sowie der Flächenpressung ermittelt werden. Für den Kühlölvolumenstrom konnte analog zum Reibungszahlverhalten, wie in [12] und [14] festgehalten, kein signifikanter Einfluss festgestellt werden. Das Kühlöl bzw. der Schmierstoff selbst nimmt hingegen einen maßgeblichen Einfluss auf das Reibverhalten im Friktionskontakt [15]. Auch nach [16] gehört das tribologische System zu einer wesentlichen Einflussgröße auf das Reibverhalten im nasslaufenden Kupplungssystem. Es sind bisher allerdings keine Untersuchungen dazu bekannt, welchen Einfluss der Schmierstoff oder auch weitere Komponenten des tribologischen Systems, auf das Schwingungsentkopplungsverhalten im Friktionskontakt haben. Hier sollen die Untersuchungen dieses Beitrags anknüpfen. Es soll auch die in [13] ermittelte Korrelation des Reibungszahlverhaltens mit der Schwingungsentkopplung im Friktionskontakt weiter untersucht werden. Dabei sind insbesondere die minimalen bzw. maximalen Reibungszahlen sowie der Reibungszahlgradient, wie in [13] definiert, von Bedeutung. Ein negativer Reibungszahlgradient kann selbsterregte Rupfschwingungen hervorrufen [17]. Auch kann ein stark positiver Reibungszahlgradient die Funktionserfüllung im Kupplungssystem negativ beeinflussen. Grundsätzlich ist daher ein leicht positiver Reibungszahlgradient bei der Auslegung des Friktionssystems erwünscht $[17,18]$. Ob eine, hinsichtlich des Reibungszahlgradienten und der Reibungszahlen gezielter abgestimmte Auslegung des Tribosystems das Schwingungsentkopplungsverhal- ten zusätzlich begünstigen kann, soll eines der Ziele der folgenden Untersuchungen sein.

\section{Validierungsumgebung und Vorgehensweise}

In Anlehnung an [19] besteht das tribologische System einer nasslaufenden Kupplung aus den Reiblamellen und den Stahllamellen, wie in Abb. 1 exemplarisch dargestellt, sowie aus dem Schmierstoff und einem Belastungskollektiv. Die Untersuchungen werden mittels der in $[8,20]$ vorgestellten Validierungsumgebung durchgeführt. In Abb. 2 ist der Aufbau schematisch dargestellt. Die Validierungsumgebung ermöglicht Untersuchungen des Schwingungsentkopplungs- sowie des Reibverhaltens im nasslaufenden Lamellenpaket unter dynamischer Anregung. Dabei werden insbesondere die triebstrangdynamischen Wechselwirkungen mit dem Fahrzeugrestsystem vereinfacht abgebildet und berücksichtigt. Die zugehörige Modellbildung wird detailliert in [21] vorgestellt. Es wird in physischer Form am Ausgang des Lamellenpakets (Massenträgheit $\mathrm{J}_{1}$ ) eine Torsionswelle mit entsprechenden Massenträgheiten ( $\mathrm{J}_{2}$ und $\mathrm{J}_{3}$ ) und Steifigkeiten $\left(\mathrm{c}_{\mathrm{i}}\right)$ eines vereinfachten konventionellen Restantriebsstrangs angebunden. $\mathrm{J}_{4}$ liegt dabei in Teilen physisch und virtuell vor. Die virtuelle Komponente ermöglicht z. B. die Abbildung verschiedener Lastzustände. Abb. 3 zeigt die Detailansicht der Prüfkammer mit entsprechenden Messelementen. Neben der Axialkraft (Klemmund Reaktionskraft) wird in erster Linie das Drehmoment am Ausgang des Lamellenpakets sowie die Drehzahl für die weitere Auswertung herangezogen. Aufgrund der hohen Steifigkeit des Aufbaus zwischen Ausgang des Lamellenpaketes und Drehzahlsensor, kann die durch den Sensor gemessene Drehzahl näherungsweise als anliegende Drehzahl am Ausgang des Lamellenpakets betrachtet werden.

Die Versuche werden in Dauerschlupfzuständen durchgeführt. Hierzu wird zu Beginn ein Grundschlupf durch eine Drehzahldifferenz an An- und Abtriebsseite eingestellt. Im

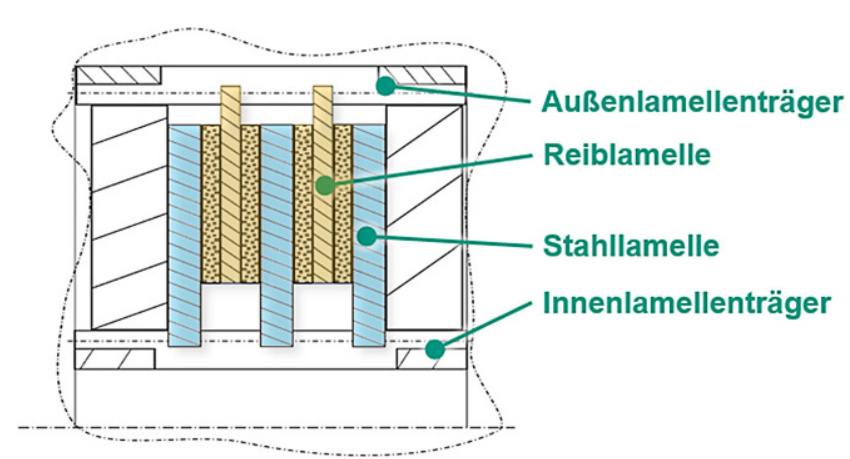

Abb. 1 Aufbau eines Lamellenpakets einer nasslaufenden Kupplung (exemplarisch) [8] 
Abb. 2 Aufbau der Validierungsumgebung, nach [20]

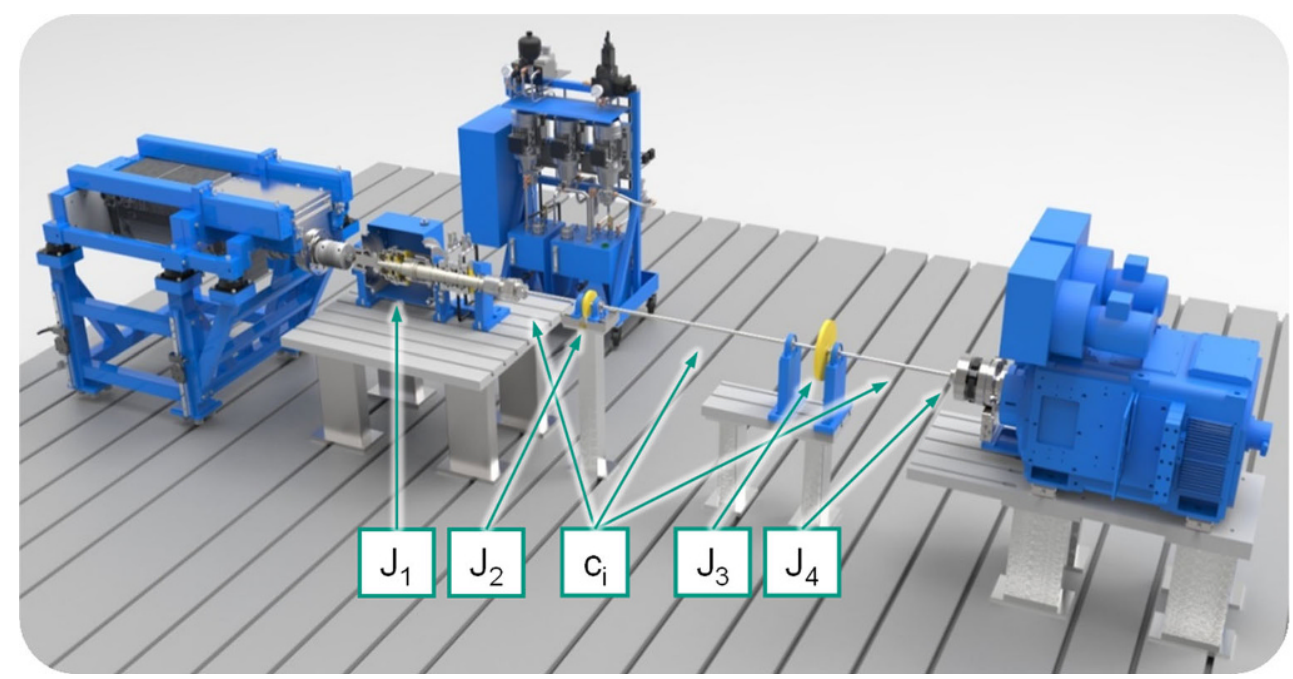

Abb. 3 Detailansicht der Prüfkammer in der Validierungsumgebung, nach [20]

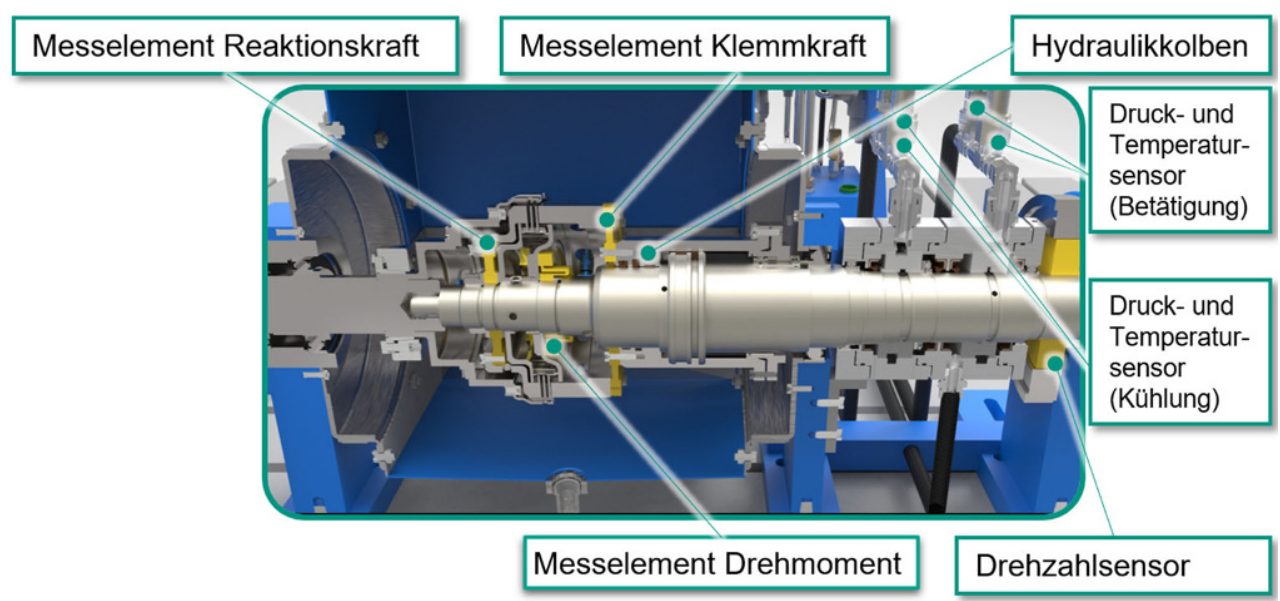

weiteren Verlauf wird antriebseitig (Eingang des Lamellenpakets, in Abb. 2 links von $\mathrm{J}_{1}$ ) eine sinusförmige Anregung aufgebracht und damit die Antriebsdrehzahl überlagert. Im eingeschwungenen dynamischen Dauerschlupfzustand werden die zuvor genannten Messgrößen erhoben. Ein Einzelversuch entspricht dabei einer Parameterkombination der Beanspruchungsgrößen, welche im weiteren Verlauf vorgestellt werden. Die Erhebung und Auswertung der Messgrößen erfolgt im eingeschwungenen Zustand über einen Bereich von $>100$ Perioden der aufgebrachten Anregung. Eine exemplarische Darstellung eines Dauerschlupfzustandes bei betätigter Kupplung und aufgebrachter Anregung ist in Abb. 4 dargestellt. Weiterführende Beschreibungen hierzu finden sich u.a. in $[8,11,12,21]$.

Um den Einfluss einer veränderten Systemstruktur in Form einer Veränderung des Tribosystems auf die Schwingungsentkopplung zu ermitteln, werden drei verschiedene Tribosystemvarianten untersucht. Es werden zum einen das Öl und zum anderen die Reiblamellen variiert. Als Kühlöl wird ein, hinsichtlich des Reibungszahlgradien-

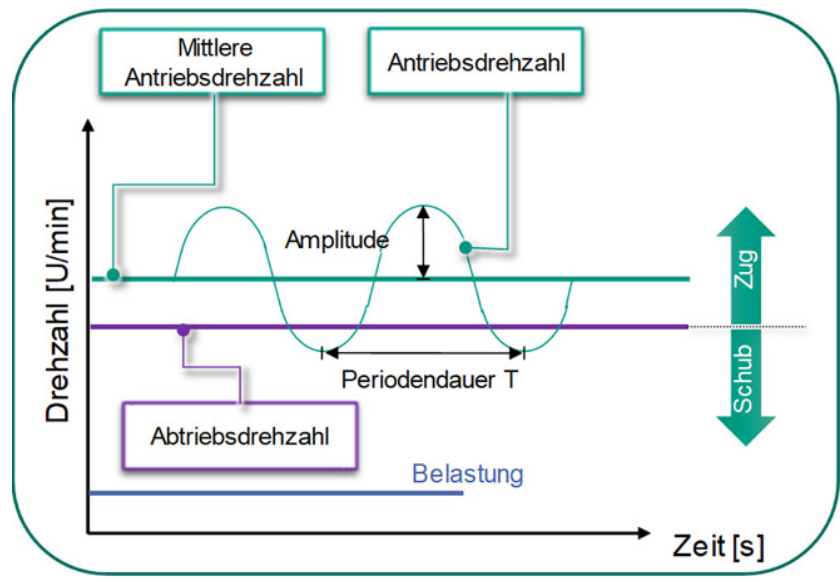

Abb. 4 Exemplarische Darstellung eines Dauerschlupfzustandes, nach [11] 
Tab. 1 Untersuchte Tribosystemvarianten

\begin{tabular}{lll}
\hline & Ölvariante & Reiblamelle \\
\hline Tribosystemvariante 1 & Typ 1 & Typ 1 \\
Tribosystemvariante 2 & Typ 2 & Typ 1 \\
Tribosystemvariante 3 & Typ 2 & Typ 2 \\
\hline
\end{tabular}

ten, also der Änderungsrate der Reibungszahl über der Gleitgeschwindigkeit, modifiziertes ATF (kinematische Viskosität bei $100^{\circ} \mathrm{C}: 4,2 \mathrm{~mm}^{2} / \mathrm{s}$ ) verwendet. Das Kühlöl wird über eine Veränderung der Additivierung mit reduzierten (Typ 1) bzw. erhöhten (Typ 2) Reibungszahlgradienten gegenüber der Serienanwendung ausgeführt. Als Reiblamellen werden gängige Papierreiblamellen eines Wandlerautomatikgetriebes mit Radialnutung (Typ 1) (Baugröße $\mathrm{d}_{\mathrm{I}} / \mathrm{d}_{\mathrm{A}}=158 / 188 \mathrm{~mm}$ ) sowie dieselben Lamellen mit erhöhter Massendichte des Reibbelags (Typ 2) verwendet. Für alle Untersuchungen werden Serienstahllamellen desselben Typs verwendet. Zwischen Tribosystemvariante 1 (TS 1) und Tribosystemvariante 2 (TS 2) wird das Kühlöl (veränderte Additivierung), zwischen TS 2 und Tribosystemvariante 3 (TS 3) die Reiblamellen (veränderter Reibbelag) geändert. Tab. 1 zeigt die Zusammensetzung der variierten Komponenten der drei Tribosystemvarianten.

Die Untersuchungen werden mittels zwei vollfaktorieller DoEs, bei welchen die folgenden Beanspruchungsgrößen in mehreren Stufen variiert werden, durchgeführt. Dadurch soll der Einfluss des Tribosystems über einen breiteren Versuchsraum, also auch bei veränderten Randbedingungen wie z.B. der Belastung, ermittelt werden können. Jede Tribosystemvariante wird gemäß der beiden in Tab. 2 aufgelisteten Versuchspläne untersucht. Es werden damit für jede Variante 64 Einzelversuche bei unverändertem, festgehaltenem „Faktor“ Tribosystem durchgeführt. Ein Einzelversuch entspricht dabei einer einzelnen Parameterkombination aus einer DoE. Die gewonnenen Erkenntnisse wurden zusätzlich mit Bestätigungsversuchen gleicher Tribosystemvarianten abgesichert und konnten damit bestätigt werden.

Als Bewertungsgrößen der Schwingungsentkopplung werden zum einen die Winkelbeschleunigung am Kupplungsausgang und zum anderen das spezifische Amplitudenverhältnis der Drehzahlen herangezogen. Die beiden

Tab. 2 Versuchsplan mit Bereichen der Beanspruchungsgrößen

\begin{tabular}{lll}
\hline Beanspruchungsgröße & $\begin{array}{l}\text { Faktorbereich } \\
\text { DoE } 1\end{array}$ & $\begin{array}{l}\text { Faktorbereich } \\
\text { DoE } 2\end{array}$ \\
\hline Grundschlupfdrehzahl in min $^{-1}$ & 10 bis 20 & 30 \\
Flächenpressung in $\mathrm{N} / \mathrm{mm}^{2}$ & 0,4 bis 0,8 & 0,4 bis 0,8 \\
Anregungsordnung & 2 bis 3. & 2. bis 3. \\
Anregungsamplitude in min $^{-1}$ & 10 & 10 bis 25 \\
Antriebsdrehzahl in min $^{-1}$ & 600 bis 1200 & 600 bis 1200 \\
$\begin{array}{l}\text { Kühlölvolumenstrom in } \\
\text { mm }^{3} /\left(\text { mm }^{2} \text { s }\right)\end{array}$ & 3 bis 6,5 & 3 bis 6,5 \\
\hline
\end{tabular}

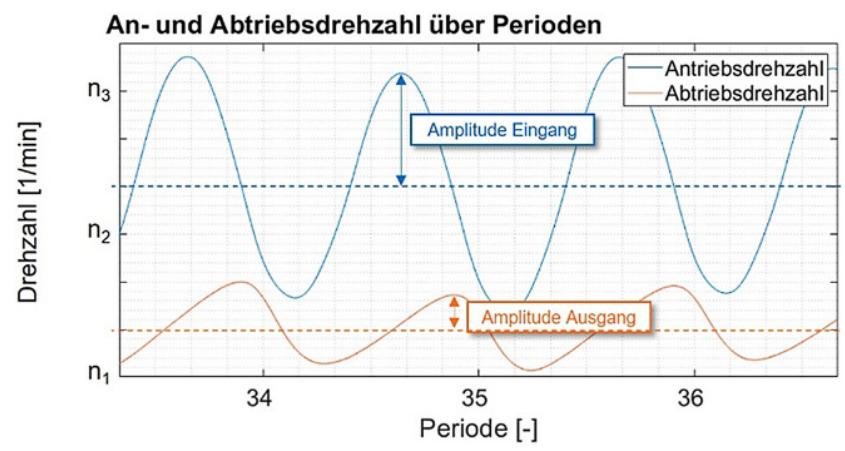

Abb. 5 Exemplarisch, Drehzahlamplituden am Kupplungsausgang und -eingang zur Ermittlung des Amplitudenverhältnisses

mitunter aus der Systemausgangsgröße Abtriebsdrehzahl ermittelten Bewertungsgrößen ermöglichen einen relativen Vergleich der Entkopplungswirkung mit anderen schwingungsreduzierenden Systemen. Das spezifische Amplitudenverhältnis wird aus den gemittelten Amplituden der Drehzahl am Kupplungsausgang und der Drehzahl am Kupplungsreingang $A V=\widehat{n}_{\text {Out }} / \widehat{n}_{\text {In }}$ bezogen auf den Frequenzbereich der gezielt aufgebrachten Anregung bestimmt. In Abb. 5 ist ein beispielhafter Verlauf der Drehzahlen für einen Dauerschlupfzustand mit einem $A V<1$ dargestellt.

\section{Dynamisches Reibungszahlverhalten}

In folgendem Abschnitt wird das dynamische Reibungszahlverhalten der verschiedenen Tribosysteme gegenübergestellt und grundlegend miteinander verglichen. Hierfür wird der nach Gl. 1 ermittelte Reibungszahlgradient $\mu^{\prime}$ sowie die in Abb. 6 exemplarisch dargestellte minimale $\left(\mu_{\min }\right)$ und maximale $\left(\mu_{\max }\right)$ Reibungszahl herangezogen [13].

$\mu^{\prime}=\frac{\mu_{\Delta n_{\max }}-\mu_{\Delta n_{\min }}}{\Delta n_{\max }-\Delta n_{\min }}$

Abb. 7 zeigt exemplarisch den Reibungszahlverlauf über der Differenzdrehzahl eines Einzelversuchs für die Tribosystemvarianten TS 1 und TS 2. Dabei werden alle Randbedingungen abgesehen des Tribosystems, hier im Speziellen

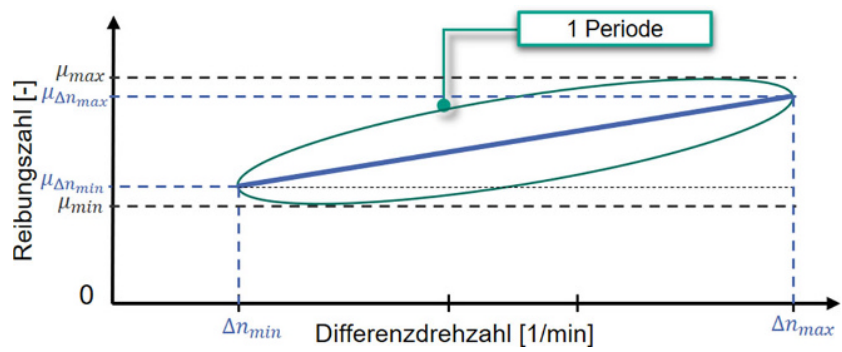

Abb. 6 Exemplarische Darstellung eines Reibungszahlverlaufs mit Bewertungsgrößen des Reibverhaltens [13] 
a

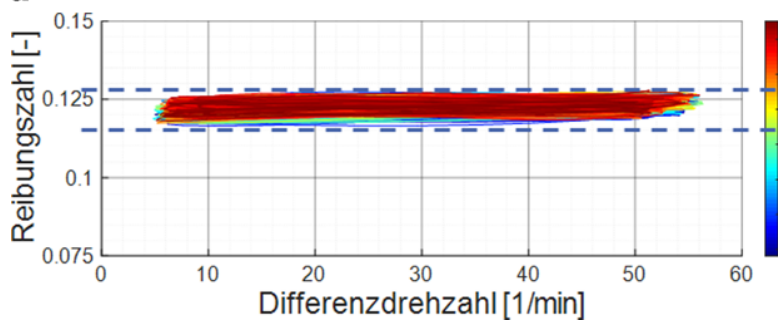

b

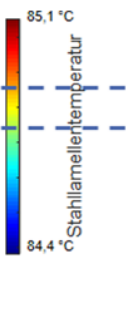

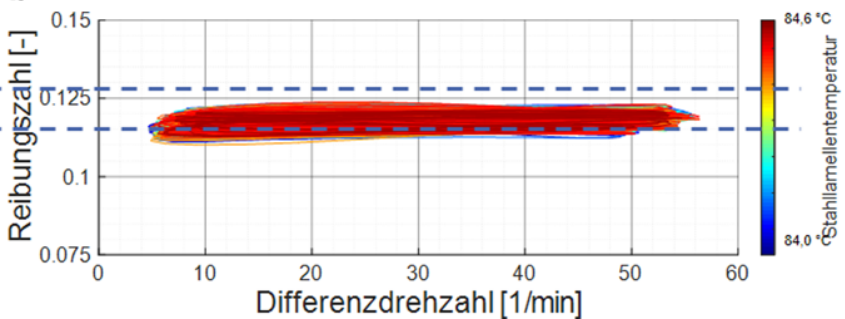

Abb. 7 Exemplarische Darstellung des Einfluss des Schmierstoffs auf die Reibungszahlen. a TS 1; b TS 2

des Schmierstoffs, gleich gehalten. Die zusätzlich aufgeführten Stahllamellentemperaturen dienen hier der Überwachung, dass die Einzelversuche aller Tribosystemvarianten in annähernd gleichen Temperaturbereichen erfolgen und werden im Rahmen dieser Untersuchung nicht tiefergehend berücksichtigt. Es wird ersichtlich, dass die Veränderung des Kühlöls hier tendenziell zu einer Abnahme der absoluten Reibungszahlen führt.

Um die ermittelte Tendenz für den vollständigen Versuchsraum verifizieren oder falsifizieren zu können, werden alle Einzelversuche des Verschsraums der jeweiligen Tribosystemvariante herangezogen. Abb. 8 zeigt dazu die Boxplots der jeweiligen maximalen Reibungszahl (a) sowie der minimalen Reibungszahl (b) aller Einzelversuche beider Tribosystemvarianten im Vergleich. Die Boxplots stellen die Auswertung der Reibungszahlen über die 64 angefahrenen Paramterkombinationen dar.

Durch die in z.B. [12] ermittelten Einflüsse der Beanspruchungsgrößen variieren die Reibungszahlen der einzelnen Versuche einer Tribosystemvariante in sich. Über alle 64 Einzelversuche einer Tribosystemvariante wurde jedoch der Faktor „Tribosystem“ konstant gehalten, so dass aus dem Vergleich des jeweils linken mit dem jeweils rechten Boxplot ein tendenzieller Einfluss des Tribosystems auf die absoluten Reibungszahlen abgeleitet wird. Die Variation des Schmierstoffs von TS 1 zu TS 2 führt tendenziell zu einer Abnahme der sowohl maximalen als auch der minimalen Reibungszahl. Es lässt sich daraus ein Einfluss des Schmierstoffs auf das Reibungszahlverhalten, im Detail betrachtet auf die absoluten Reibungszahlen im Friktionskontakt, festhalten.
Abb. 8 Einfluss des Schmierstoffs - Boxplot der maximalen (a) und minimalen Reibungszahlen (b) aller Einzelversuche für TS 1 und TS 2
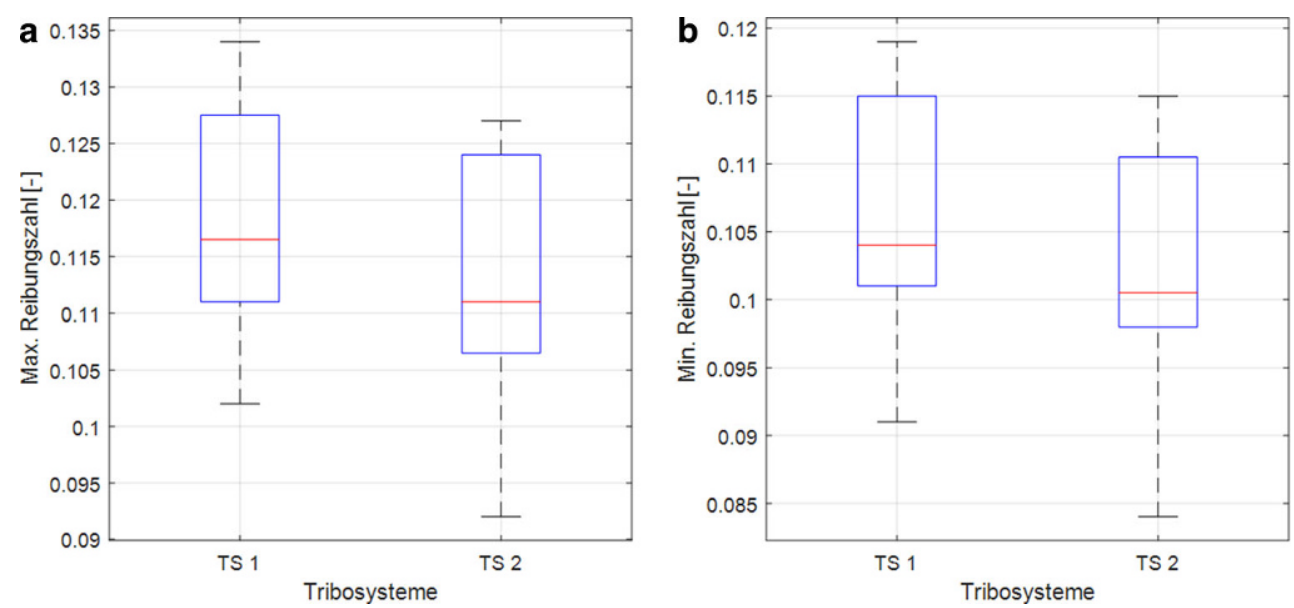

a

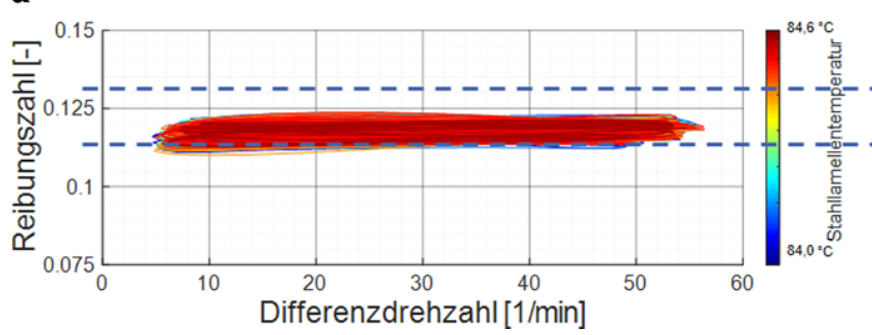

b

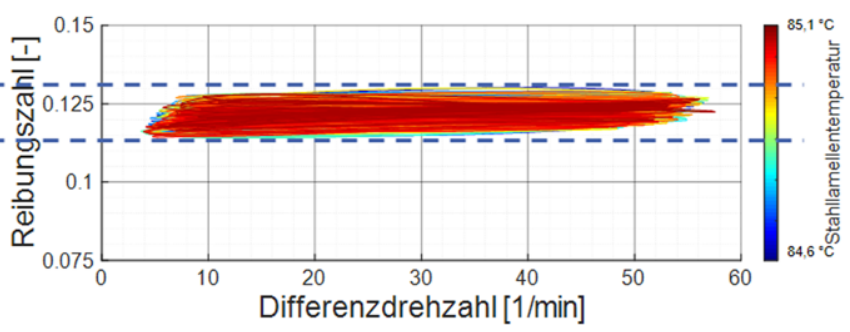

Abb. 9 Exemplarische Darstellung des Einfluss der Reiblamelle auf die Reibungszahlen. a TS 2; b TS 3 
Abb. 10 Einfluss des Reibbelags - Boxplot der maximalen (a) und minimalen Reibungszahlen (b) aller Einzelversuche für TS 2 und TS 3

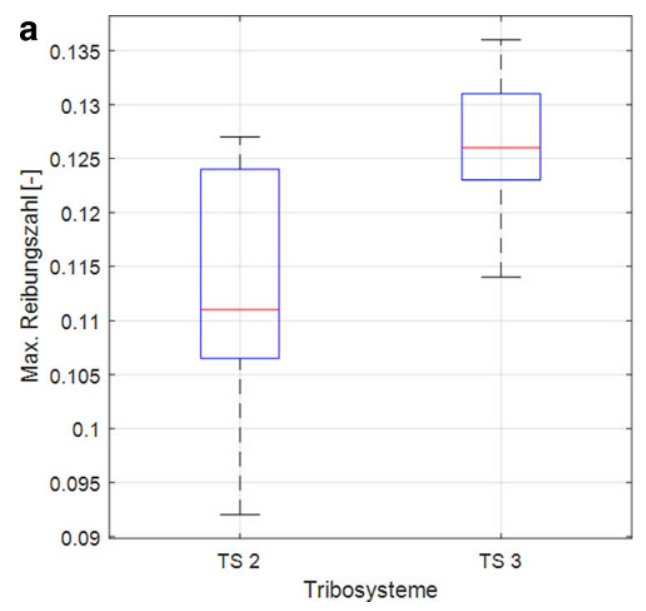

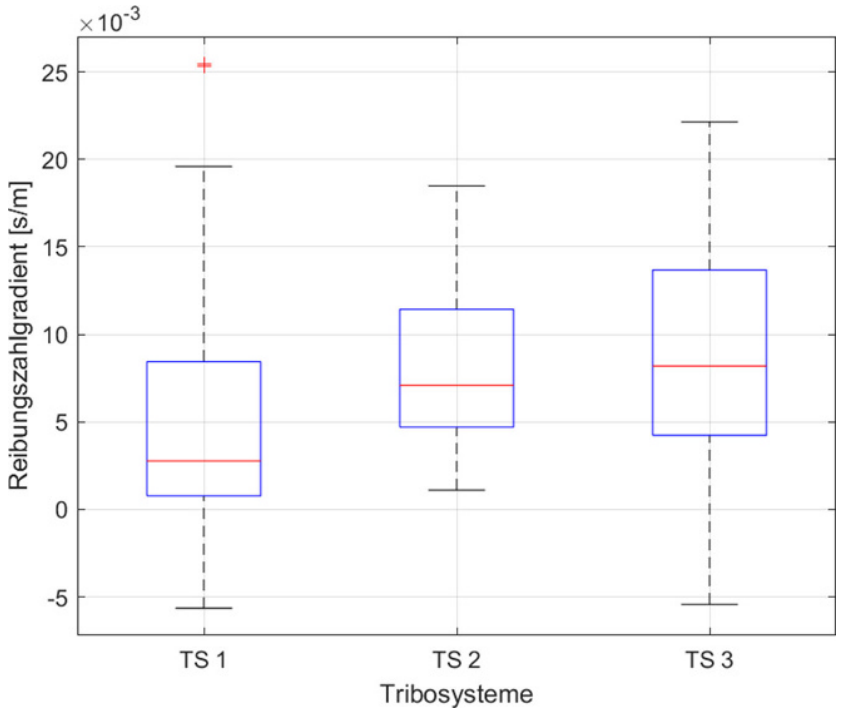

Abb. 11 Einfluss des Schmierstoffs und des Reibbelags - Boxplot der Reibungszahlgradienten aller Einzelversuche für TS 1-TS 3

In Abb. 9 ist exemplarisch der Reibungszahlverlauf eines Einzelversuchs für die Variation des Reibbelags durch TS 2 und TS 3 dargestellt. Die Veränderung des Tribosystems führt hier demnach zu tendenziell höheren Reibungszahlwerten.

Zur näheren Betrachtung werden im Folgenden wieder die Boxplots der Reibungszahlen aller Einzelversuche verglichen. Aus Abb. 10 wird eine Erhöhung der absoluten Reibungszahl bei Veränderung des Reibbelags von TS 2 zu TS 3 hin ersichtlich. Der Reibbelag kann damit ebenso wie der zuvor betrachtete Schmierstoff als ein Einflussfaktor auf das Reibungszahlverhalten, im Detail auf die absoluten Reibungszahlen im Friktionskontakt identifiziert werden.

Abschließend wird der Einfluss auf den Reibungszahlgradienten betrachtet. In Abb. 11 lässt sich durch die Erhöhung des Median eine tendenzielle Erhöhung des Gradienten durch die Variation des Schmierstoffs von TS 1 zu TS 2 und TS 3 erkennen. Ein maßgeblicher Einfluss des Reibbe- lags auf den Median des Reibungszahlgradienten lässt sich im untersuchten Versuchsraum von TS 2 zu TS 3 hingegen nicht erkennen. Es lässt sich daher keine Aussage über eine signifikante $\mathrm{Zu}$ - oder Abnahme des Gradienten treffen. Durch die Veränderung des Reibbelags von TS 2 zu TS 3 wird die Range des Reibungszahlgradienten im Untersuchungsraum allerdings analog zu TS 1 bis hin zu negativen Gradienten, vergrößert.

Zusammenfassend kann festgehalten werden, dass ein Einfluss des Schmierstoffs auf den Reibungszahlgradienten, insbesondere auf die Absolutwerte dessen, vorliegt. Die hier vorgenommene Veränderung der Additivierung des Kühlöls führt tendenziell zu einer Erhöhung des Reibungszahlgradienten.

\section{Schwingungsentkopplungsverhalten}

Mittels der in Kap. 2 vorgestellten relativen Bewertungsgrößen wird in folgenden Abschnitten ein relativer Einfluss des tribologischen Systems auf das Schwingungsentkopplungsverhalten im nasslaufenden Lamellenpaket aus den durchgeführten Untersuchungen herausgearbeitet. Daran anknüpfend wird eine mögliche Korrelation der Schwingungsentkopplung mit dem Reibungszahlverhalten untersucht. In nachfolgender Abb. 12 wird exemplarisch für eine Parameterkombination (Einzelversuch), also gleichbleibenden Randbedingungen, insbesondere gleichbleibender Anregung, die An- (blau) sowie die Abtriebsdrehzahl (orange) am Eingang bzw. Ausgang des Lamellenpakets für TS 1 und TS 2 aufgetragen. Die Abtriebsdrehzahl weist hier bei TS 2 grundsätzlich gegenüber TS 1 höhere Amplituden auf, was bei gleicher Anregungsamplitude und Anregungsfrequenz zu einem höheren Amplitudenverhältnis sowie einer erhöhten Winkelbeschleunigung bei TS 2 führt. Bestätigt wird die graphische Auswertung durch die zugehörigen Kennwerte in Tab. 3. Diese werden entsprechend der Ausführung in 


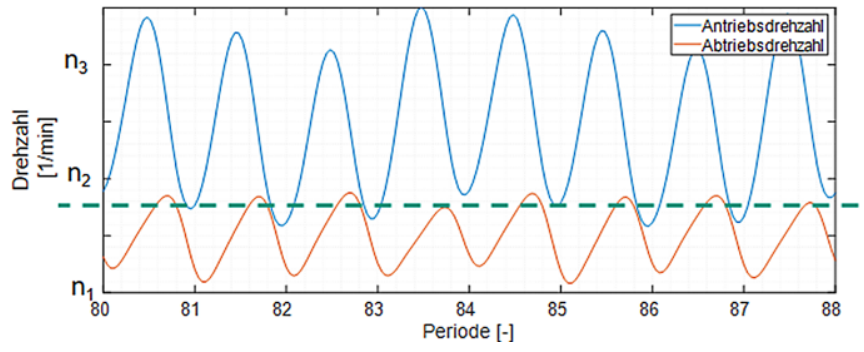

b

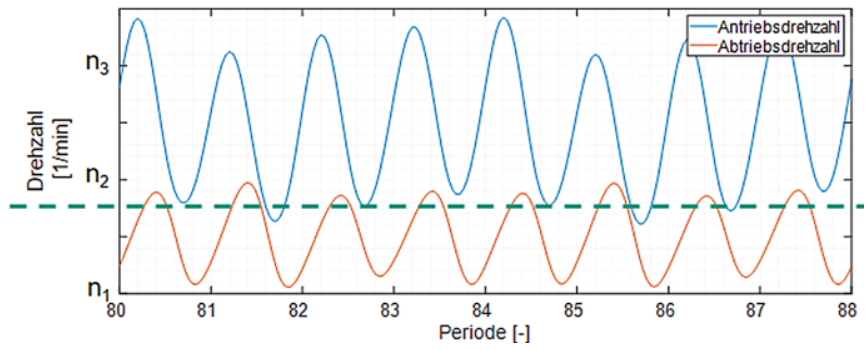

Abb. 12 Exemplarische Darstellung des Einfluss des Schmierstoffs auf die Schwingungsentkopplung. a TS 1; b TS 2

Tab. 3 Schwingungsentkopplungsbewertungsgrößen zu Abb. 12 Einfluss des Schmierstoffs

\begin{tabular}{lll}
\hline & TS 1 & TS 2 \\
\hline Spez. Amplitudenverhältnis [\%] & 42,8 & 52,8 \\
Winkelbeschleunigung [rad/s $\left.\mathrm{s}^{2}\right]$ & 179 & 212 \\
\hline
\end{tabular}

Kap. 2 gemittelt über den jeweiligen Dauerschlupfzustand bestimmt.

Die in Abb. 13 abgebildeten Boxplots des spezifischen Amplitudenverhältnisses sowie der Winkelbeschleunigung aller Einzelversuche der beiden Tribosysteme TS 1 und TS 2 sowie die in Abb. 14 abgebildeten zugehörigen Ab- solutwerte des spezifischen Amplitudenverhältnis für jeden Einzelversuch derselben Parameterkombinationen für TS 1 (orange) und TS 2 (blau) bestätigen den Einfluss des Schmierstoffs auf die Schwingungsentkopplung im nasslaufenden Friktionskontakt.

Der Median des Amplitudenverhältnisses ist bei TS 2 um etwa (absolut) 14\% erhöht. Durch Veränderung der Additivierung des Kühlöls von TS 1 zu TS 2 lässt sich somit eine deutliche Abnahme der Schwingungsentkopplungswirkung erkennen. Der Schmierstoff kann als ein Einflussfaktor auf die Schwingungsentkopplung festgehalten werden.
Abb. 13 Einfluss des Schmierstoffs - Boxplot des spez. Amplitudenverhältnis (a) und der Winkelbeschleunigung (b) aller Einzelversuche für TS 1 und TS 2
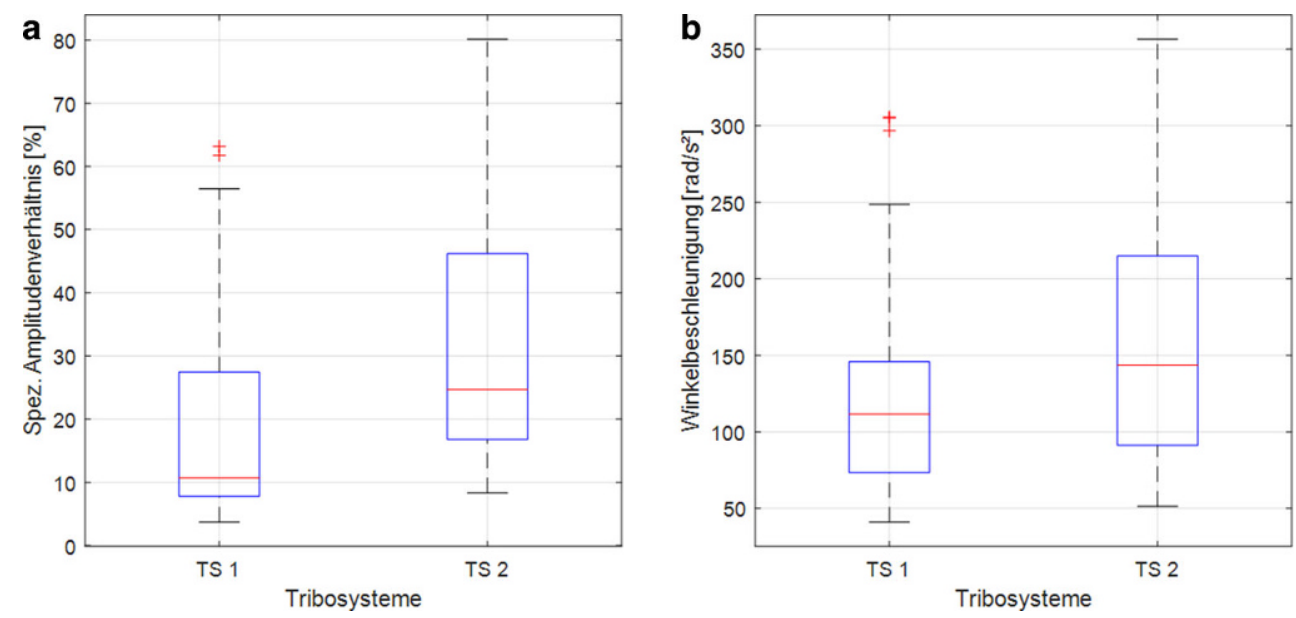

Abb. 14 Einfluss des Schmierstoffs - Verlauf des spez. Amplitudenverhältnis über alle Einzelversuche für TS 1 (orange) und TS 2 (blau)

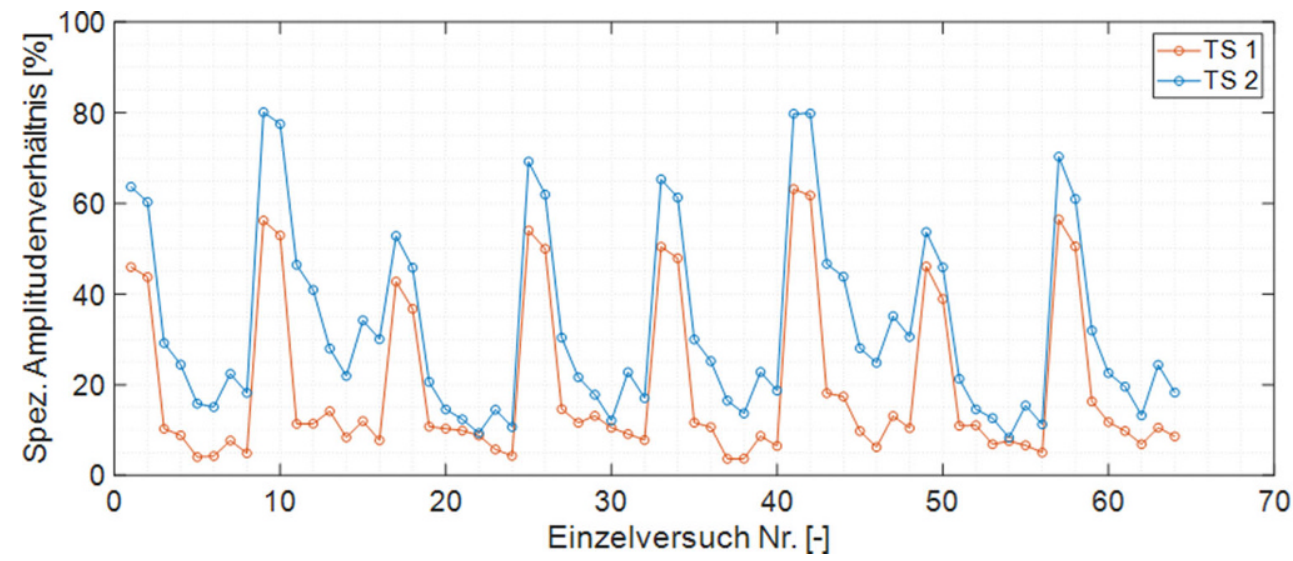


Abb. 15 Einfluss des Reibbelags - Boxplot des spez. Amplitudenverhältnis (a) und der Winkelbeschleunigung (b) aller Einzelversuche für TS 2 und TS 3

Abb. 16 Einfluss des Reibbelags - Verlauf des spez. Amplitudenverhältnis über alle Einzelversuche für TS 2 (blau) und TS 3 (orange)
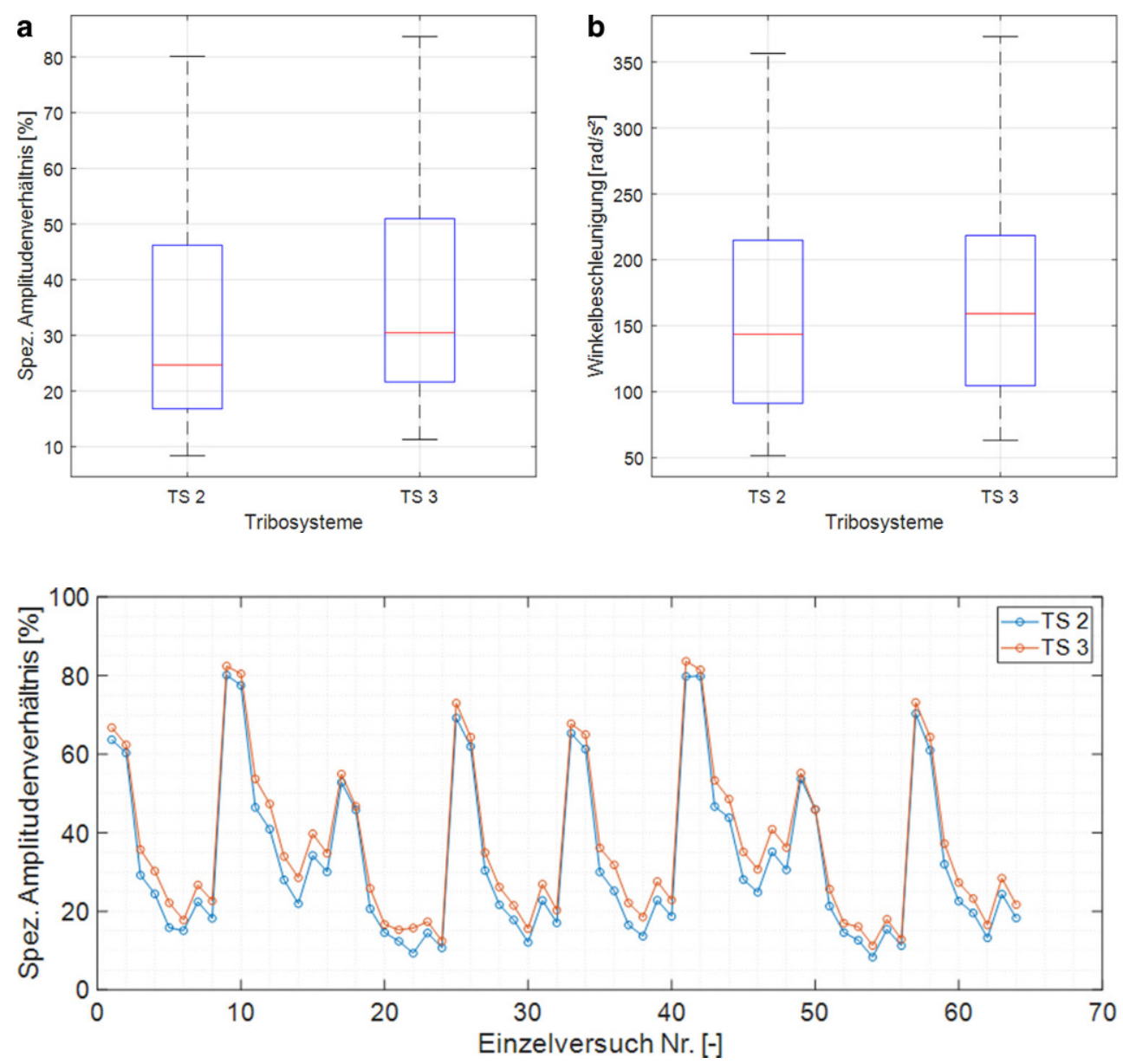

In Abb. 15 und Abb. 16 werden die Ergebnisse der Untersuchung des Einfluss des Reibbelags auf die Schwingungsentkopplung dargestellt. Analog zur Darstellung des Einfluss des Schmierstoffs, zeigt Abb. 15 die Boxplots aller Einzelversuche von TS 2 und TS 3. Abb. 16 zeigt ergänzend die zugehörigen Absolutwerte des spezifischen. Amplitudenverhältnis für jeden Einzelversuch.

Sowohl das spezifische Amplitudenverhältnis (Abb. 15a) als auch die Winkelbeschleunigung (Abb. 15b) nehmen mit Veränderung des Reibbelags von TS 2 zu TS 3 zu. Über alle Einzelversuche liegt der Median des Amplitudenverhältnisses bei TS 3 um (absolut) 6\% höher. Verdeutlicht wird dies auch durch den Verlauf der Absolutwerte des Amplitudenverhältnisses von TS 3, dessen Kurve einen leicht höheren Verlauf gegenüber der Kurve von TS 2 aufweist (Abb. 16). Auch durch den veränderten Reibbelag lässt sich daher ein Einfluss auf die Schwingungsentkopplung ermitteln.

\section{Zusammenfassung und Ausblick}

Es werden Ergebnisse zur Untersuchung des Einflusses des tribologischen Systems auf das Reibungszahlverhal- ten sowie die Schwingungsentkopplung im nasslaufenden Friktionskontakt unter dynamischer Anregung vorgestellt. Die Untersuchungen werden unter Berücksichtigung triebstrangdynamischer Wechselwirkungen mit dem Fahrzeugrestsystem anhand zweier Öl- sowie zweier Reiblamellenvarianten durchgeführt. Zusammenfassend kann festgehalten werden, dass sowohl der Schmierstoff bzw. das Kühlöl als auch der Reibbelag einen Einfluss auf das Reibungszahl- sowie auf das Entkopplungsverhalten haben. Dabei wird bei der Variation der Öladditivierung von TS 1 zu TS 2:

- eine Zunahme des Reibungszahlgradienten,

- eine Abnahme der absoluten Reibungszahlen,

- eine Abnahme der Schwingungsentkopplungswirkung,

bei der Variation des Reibbelags von TS 2 zu TS 3:

- eine Erweiterung der Range des Reibungszahlgradienten hin zu negativen Gradienten,

- eine Zunahme der absoluten Reibungszahlen,

- eine Abnahme der Schwingungsentkopplungswirkung

ermittelt. 
Eine tendenzielle Korrelation des Reibungszahlgradienten mit der Entkopplung kann daraus abgeleitet werden. Im untersuchten Bereich führt eine Zunahme des Gradienten tendenziell zur Abnahme der Entkopplungswirkung und damit zu einer tendenziellen Verschlechterung des Komfortverhaltens. Zwischen den absoluten Reibungszahlen und der Entkopplungswirkung kann im untersuchten Bereich hingegen kein grundsätzlicher Zusammenhang festgestellt werden.

Es wird außerdem ein maßgeblicher Einfluss des Tribosystems (Schmierstoff und Reibbelag) auf die Schwingungsentkopplungswirkung im nasslaufenden Lamellenpaket ermittelt. Eine gezielte Auslegung des Tribosystems kann die Schwingungsreduzierung begünstigen und die nasslaufende Kupplung als mögliches Stellglied hierfür befähigen. Die Variation des Tribosystems kann dabei auch Einfluss auf die Dämpfungswirkung nehmen und möglicherweise andere schwingungsmindernde Mechanismen begünstigen. Eine dadurch ermöglichte Effizienzsteigerung kann zusätzlich zu einer günstigeren Auslegung beitragen, weswegen zukünftige Untersuchungen daran anknüpfend auch den Zielkonflikt Komfort und Effizienz aufgreifen sollen.

Danksagung Die Autoren danken für die Unterstützung des Forschungsprojekts. Das IGF-Vorhaben 18501-N der Forschungsvereinigung Antriebstechnik e. V. (FVA) wird über die AiF im Rahmen des Programms zur Förderung der Industriellen Gemeinschaftsforschung (IGF) vom Bundesministerium für Wirtschaft und Energie aufgrund eines Beschlusses des Deutschen Bundestages gefördert.

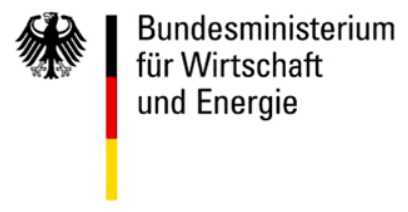

Funding Open Access funding enabled and organized by Projekt DEAL.

Open Access Dieser Artikel wird unter der Creative Commons Namensnennung 4.0 International Lizenz veröffentlicht, welche die Nutzung, Vervielfältigung, Bearbeitung, Verbreitung und Wiedergabe in jeglichem Medium und Format erlaubt, sofern Sie den/die ursprünglichen Autor(en) und die Quelle ordnungsgemäß nennen, einen Link zur Creative Commons Lizenz beifügen und angeben, ob Änderungen vorgenommen wurden.

Die in diesem Artikel enthaltenen Bilder und sonstiges Drittmaterial unterliegen ebenfalls der genannten Creative Commons Lizenz, sofern sich aus der Abbildungslegende nichts anderes ergibt. Sofern das betreffende Material nicht unter der genannten Creative Commons Lizenz steht und die betreffende Handlung nicht nach gesetzlichen Vorschriften erlaubt ist, ist für die oben aufgeführten Weiterverwendungen des Materials die Einwilligung des jeweiligen Rechteinhabers einzuholen.

Weitere Details zur Lizenz entnehmen Sie bitte der Lizenzinformation auf http://creativecommons.org/licenses/by/4.0/deed.de.

\section{Literatur}

1. Albers A (1990) Elektronisches Kupplungsmanagement (EKM) Die mitdenkende Kupplung. 4. Internationales Kolloquium Torsionsschwingungen im Antriebsstrang: 4. Internationales Kolloquium am 20. April 1990 in Baden-Baden, LuK, S. 77-101

2. Lutz D, Deutscher Ingenieure V (1988) Kupplungsmanagement ein Baustein zur Drehschwingungsdämpfung. Berichte, Bd. 697. VDI-Verlag, Düsseldorf, S 219-256

3. Jürgens G, Fischer R (1988) Vergleich verschiedener Systeme zur Verringerung von Triebstrangschwingungen, S 233-256

4. Drexl H-J, Deutscher Ingenieure V (1988) Der Torsionsdämpfer in der Kupplungsscheibe. Berichte, Bd. 697. VDI-Verlag, Düsseldorf, S $133-158$

5. Reik W, Friedmann O, Agner I, Werner O (2004) Die Kupplung das Herz des Doppelkupplungsgetriebes. In Getriebe in Fahrzeugen 2004. Tagung Friedrichshafen, 22. und 23. Juni 2004. VDI-Berichte, 1827, Buch. Düsseldorf: VDI, S 65-88

6. Abbassi MB (1999) Steigerung des Antriebsstrangkomforts im Kfz durch elektronisches. Kupplungsmanagement Atz - Automob Z 101(2):118-126

7. Albers A, Ott S, Basiewicz M (2017) Influencing factors on the decoupling of induced rotational oscillation by wet-running multiplate-packages in controlled slip mode. In: 44. Leeds-Lyon Symposium on Tribology. Lyon. https://leeds-lyon2017.sciencesconf.org/ 140721/document

8. Albers A, Ott S, Basiewicz M (2017) Ermittlung des Übertragungs- und Systemverhaltens nasslaufender Lamellenpakete im Schlupfbetrieb unter Berücksichtigung der Systemwechselwirkungen. Kupplungen und Kupplungssysteme in Antrieben. Ettlingen. VDI-Verlag, Düsseldorf

9. Vorholt S (2011) Untersuchung und Simulation von Reibschwingungen an einer Wandlerüberbrückungskupplung. Schriftenreihe. Institut: Product and Service Engineering (11.1), Ruhr-Universität Bochum, Universitätsbibliothek Doctoralthesis, Bochum

10. Mosbach C (2002) Untersuchung der Ursachen von Reibschwingungen und Geräuschen an ölgekühlten Reibschaltelementen. Abschlußbericht; Forschungsvorhaben Nr. 297 Reibschwingungen. Forschungsheft, Forschungsvereinigung Antriebstechnik, Bd. 650. FVA, Frankfurt

11. Kemper C, Basiewicz M, Ott S, Albers A (2018) Schwingungsentkopplung durch nasslaufende Funktionsreibkontakte im geregelten Schlupfbetrieb - Anforderungen an den tribologischen Kontakt in Wechselwirkung mit der Antriebssystemdynamik. 59. TribologieFachtagung 2018. Göttingen

12. Kemper C, Wantzen K, Bischofberger A, Ott S, Albers A (2019) Vergleich von Reibungszahlverläufen von nasslaufenden Friktionskontakten im gezielten Schlupfbetrieb unter dynamischer Anregung. Forschung im Ingenieurwesen 83(2):239-246

13. Bischofberger A, Ott S, Albers A (2019) Einfluss von Beanspruchungsgrößen auf die Schwingungsentkopplung im nasslaufenden Kupplungssystem und deren Korrelation mit dem Reibverhalten im Friktionskontakt. 60. Tribologie-Fachtagung 2019. Göttingen

14. Rank R (2002) Reibwerkstoffe und Systemlösungen für neue KfZGetriebe. Lubricants, Materials and Lubrication. Engineering, Technische Akademie Esslingen, Ostfildern, S 1925-1936

15. Höhn B-R, Pflaum H, Mosbach C (2003) Methodik zur Beurteilung des Schmierstoffeinflusses auf das Reibschwingverhalten nasslaufender Lamellenkupplungen. VDI Ber 1786:455-468

16. Wienholt HW, Zdora D (1995) Auslegung der Überbrückungskupplung mit geregeltem Schlupf bei Einsatz des Drehmomentwandlers in Pkw-Automatikgetrieben, S 281-299

17. Albers A, Herbst D (1998) Kupplungsrupfen - Ursachen, Modellbildung und Gegenmaßnahmen. VDI-Berichte 1416; ISBN 3-18091416-5 
18. Kani H, Miyake J, Ninomiya T (1992) Analysis of the Friction Surface on Clutch Judder. Technical Notes 13.1:82-84

19. GfT (2003) Arbeitsblatt, Bd. 7. Tribologie Verschleiß, Reibung Definitionen, Begriffe, Prüfung, Moers

20. Ott S, Basiewicz M (2017) Innovative validation environments and methods for holistic clutch system development. Antriebstechnisches Kolloquium ATK 2017. Tagungsband 17:12
21. Ott S, Basiewicz M (2017) Schwingungsreduzierung durch das Kupplungssystem im gezielten Schlupfbetrieb. Kupplungen und Kupplungssysteme in Antrieben. Ettlingen. VDI-Verlag, Düsseldorf 